\title{
Effects of Antidepressant Withdrawal on the Dexamethasone Suppression Test
}

\author{
Steven C. Dilsaver and John F. Greden
}

Received July 23, 1984; revised version received October 1, 1984; accepted November 2, 1984.

\begin{abstract}
Withdrawal of antidepressant medications may be a source of variance in dexamethasone suppression test (DST) results. We investigated this issue retrospectively through the serial analysis of postdexamethasone plasma cortisol concentrations and clinical severity in 15 patients and 9 control subjects before, during, and after antidepressant withdrawal. Postdexamethasone cortisol concentrations were significantly higher during the antidepressant withdrawal phase. Drug-withdrawal subjects also had an increased frequency of DST nonsuppression. Recent withdrawal of antidepressarts requires further study as a possible source of variance for hypothalamic-pituitary-adrenal measures.
\end{abstract}

Key Words. Dexamethasone suppression test, neuroendocrine, antidepressants, drug withdrawal.

The performance of the dexamethasone suppression test (DST) as a diagnostic indicator is subject to many sources of variance. These include the severity of depression (Rothschild and Schatzberg, 1982; Peselow et al., 1983), presence or absence of delusions (Rothschild and Schatzberg, 1982; Rudorfer et al., 1982; Schatzberg et al., 1983), recent weight loss (Berger et al., 1982; Edelstein et al., 1982; Berger et al., 1983), differences in diagnostic patterns and operational criteria from one setting to another (Targum et al., 1982a), dose of dexamethasone (Rush et al., 1982; Brown et al., 1983), number of postdexamethasone plasma samples analyzed (Asfeldt, 1969; Carroll et al., 1981), timing of such samples (Goldberg, 1980; Sachar et al., 1983), number of DSTs performed (Carroll et al.. 1981; Greden et al., 1982), time interval between DSTs, and specificity and sensitivity of assays used to measure plasma cortisol concentrations (Demers and Derck, 1977; Meltzer, 1983; Wood et al., 1983).

We hypothesized that recent antidepressant withdrawal is a source of variance in DST results (Dilsaver et al., 1983b; Dilsaver and Greden, 1984b). We tested this hypothesis by retrospectively measuring postdexamethasone plasma cortisol concentrations and clinical severity in patients and control subjects before, during, and after antidepressant withd rawal. After formulating the hypothesis tested in this study, we noted (Greden et al., 1983) that three patients monitored with weekly DSTs had

Steven C. Dilsaver. M.D., is Senior Research Track Resident and John F. Greden, M.D., is Professor of Psychiatry and Director. Clinical Studies Unit for Affective Disorders and Senior Research Associate, Mental Health Research Institute, University of Michigan, Ann Arbor. (Keprint requests to Dr. S.C. Dilsaver, Dept. of Psychiatry, University of Michigan, Ann Arbor, MI 48109. USA.) 
suppressive (normal) responses while receiving tricyclic antidepressants but nonsuppressive (abnormal) responses soon after drug discontinuation. We conjectured that these nonsuppressive responses might be due to one of the following factors: (1) patients may have been partially treated, thus accounting for the suppressive response despite continued underlying neurobiological abnormalities that become salient only after antidepressant withdrawal; or (2) the emergence of a nonsuppressive response could be due to drug discontinuation-for example, an antidepressant (anticholinergic) withdrawal syndrome with secondary cholinergic overdrive could theoretically be a sufficient cause of DST nonsuppression (Dilsaver et al., 1983b; Dilsaver and Greden, 1984b). Physostigmine-induced cholinergic excess can produce DST nonsuppression in normal subjects who had suppressive responses at baseline (Carroll et al., 1980; Doerr and Berger, 1983). Further, antidepressant discontinuation can produce cholinergic overdrive (Dilsaver et al., 1983a, 1983b; Dilsaver and Greden, $1983,1984 b$ ), especially if the antidepressants are those with documented anticholinergic potency. Consequently, it is a distinct possibility that antidepressant withdrawal produces "false-positive" DSTs. This question can be answered definitively only with a prospective, placebo-controlled, crossover design. Nevertheless, because drug-withdrawal programs have been used in most neuroendocrine studies, this source of variance could be extremely important. Thus, as an initial exploration, we addressed this question using a retrospective data base of serial DSTs collected from patients withdrawn from medications.

\section{Methods}

Inclusion Criteria: Drug-Withdrawal Patients. Inclusion criteria for the 15 patients withdrawn from medication were as follows: (1) hospitalization on the Clinical Studies Unit (CSU) for Affective Disorders, a 12-bed research unit at the University of Michigan Medical Center; (2) diagnosis of major depressive disorder, endogenous subtype, based on Research Diagnostic Criteria (RDC) (Spitzer et al., 1977); (3) withdrawal from tricyclic antidepressants shortly before or after admission to the hospital (see Results); (4) no use of benzodiazepines or sedative-hypnotics for 1 month preceding antidepressant discontinuation; (5) no weight loss > $20 \%$ of normal body weight (for weekly weight changes, see Results); (6) no known technical exclusion factors that might interfere with the DST (Carroll et al., 1981).

Inclusion Criteria: Control Patients. To control at least partially for the stress of hospitalization. we identified nine control subjects who had been free of medication for a minimum of $9-14$ days before hospital admission. Because of the nature of the design, they could not be matched for all variables with the 15 drug-withdrawal subjects.

Operational Definitions. (1) Prewithdrawal phase: The time period before antidepressant medications were reduced in dosage or discontinued. (2) Withdrawal phase: The first 14 days following the discontinuation of antidepressants (other than monoamine oxidase inhibitors). (3) Postwithdrawal (drug-free) phase: The 15th day after receiving the last dose of antidepressants and beyond, but before starting any new pharmacological treatments.

Evaluation Procedure. Each patient received the routine CSU diagnostic evaluation, which includes the following: a 10- to 14-day drug-free period; two or three independent unstructured clinical interviews conducted by psychiatrists: a structured interview using the Schedule for Affective Disorders and Schizophrenia (Spitzer and Endicott, 1975); a diagnostic family interview conducted by a senior social worker to document longitudinal course, family history, 
and response to previous treatments; a review of past medical records whenever available to confirm clinical course; and a comprehensive physical examination and laboratory screening for medical illness. Consensus diagnoses then were formulated using Research Diagnostic Criteria (RDC) (Spitzer et al., 1977).

Clinical and demographic features are provided in Tables 1 and 2. It should be noted that four subjects ( \#2, \#5, \#10, and \#14; Table 1) also were withd rawn simultaneously from antipsychotic medications (fluphenazine, haloperidol, trifluoperazine, and chlorpromazine, respectively).

We administered $1 \mathrm{mg}$ of oral dexamethasone at $2330 \mathrm{~h}$ and collected postdexamethasone plasma cortisol samples the following day at $1600 \mathrm{~h}$ and $2300 \mathrm{~h}$. Plasma samples were assayed for cortisol using a modification of Murphy's (1967) competitive protein-binding technique. The intra-assay coefficient of variation in our laboratory is $6.5 \%$. The inter-assay coefficient of variation is $12.5 \%$ when the mean cortisol concentration is $2.5 \mu \mathrm{g} / \mathrm{dl}$ and $9.0 \%$ when it is 10 $\mu \mathrm{g} / \mathrm{dl}$. The DST was repeated weekly.

We considered every postdexamethasone cortisol sample when we categorized subjects as nonsuppressors or suppressors. Subjects were classified as nonsuppressors if the highest postdexamethasone plasma cortisol concentration exceeded $5 \mu \mathrm{g} / \mathrm{dl}$. The referent value of $5 \mu \mathrm{g} / \mathrm{dl}$ was determined in our setting only after evaluation of patients with the disease in question (endogenous depression), patients with different disease processes (psychiatric controls), and individuals with no psychiatric disease (normal controls) (Carroll et al., 1981). Our referent value is based upon application of the principles of conditional probability (Galen and Gambino, 1975) to data collected on patients hospitalized on the CSU and measurements of plasma cortisol concentration using the competitive protein binding assay of Murphy (1967). It should not be generalized indiscriminately to other settings.

Each patient's primary clinician completed the 17-item Hamilton Rating Scale for Depression (HRSD) (IIamilton, 1960) weekly. Raters were always unawate of DST results, which were unavailable to clinicians until after the patient's hospital discharge.

Data Analysis. Postdexamethasone cortisol levels were logarithmically transformed to improve normality. Paired Student's $t$ tests were used to ascertain the level of significance of the differences in cortisol concentration during the various phases of study. When applicable, nonpa rametric methods were used to measure the significance of differences in the frequency of positive DSTs under the various conditions of the study. Since clinical ratings were not normally distributed even after logarithmic transformation, we compared these data with nonparametric tests. To assess possible relationships between postdexamethasone plasma cortisol concentrations and corresponding HRSD scores, we calculated correlation coefficients. One-tailed statistical analyses were used only in those instances in which our hypotheses predicted the direction of change effected by an experimental manipulation.

Patients \#1. \#3, and \#5 (Table 1) had DSTs in all three phases, i.e., prewithdrawal, withdrawal, and post withdrawal. Patient \#15 had three DSTs, but the last two were both during the withdrawal phase. Fourteen patients, excluding \#15 (Table 1), had withdrawal and postwithdrawal (drug-free) DSTs. Based upon these distinctions, we subdivided the patient sample into two partially overlapping subsamples (\#1, \#3, \#5, and \#15, first subsample; \#1-\#14, second subsample). For each subsample, we compared cortisol concent rations from each phase with those from the other two phases using Student's paired $t$ tests.

\section{Results}

Fig. 1 presents the changes in cortisol levels between prewithdrawal, withdrawal, and postwithdrawal phases for the four subjects with DSTs in all three phases. Two of the four subjects had nonsuppressive DSTs during antidepressant treatment (prewithd rawal), while all four were nonsuppressive during the withd rawal phase. Each of the four showed a robust increase (mean $\pm \mathrm{SD}=10.2 \pm 6.8)$ in cortisol level $(t=2.87, d f=$ $3, p=0.033$, paired $t$ test, one-tailed) during withdrawal without significant change in 





寸난

盛

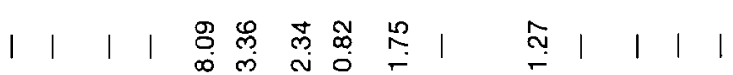

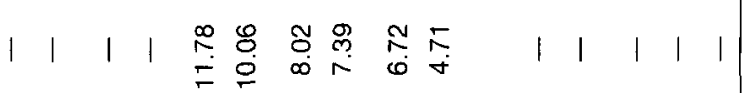

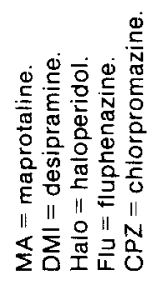

\begin{tabular}{lllllllllllllll} 
& 1 & 1 & 1 & 1 & 1 & $\underset{\sigma}{N}$ & \multirow{2}{N}{} & 1 & 1 & 1 & 1 & 1 & 1 & 1
\end{tabular}

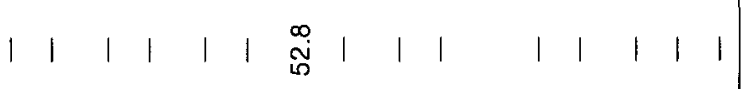

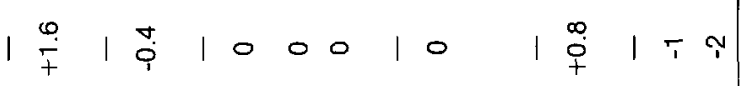

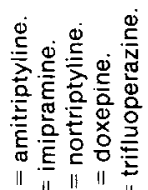
กี

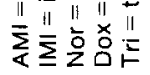

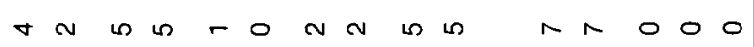

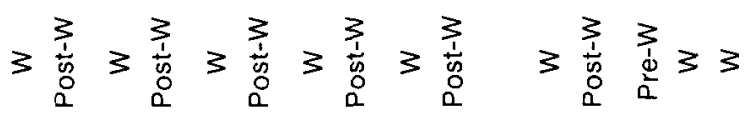

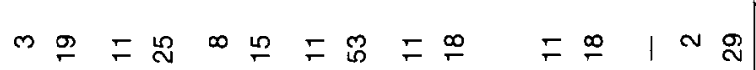

요 윤 은 융 우 은

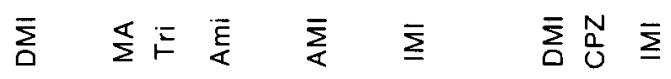

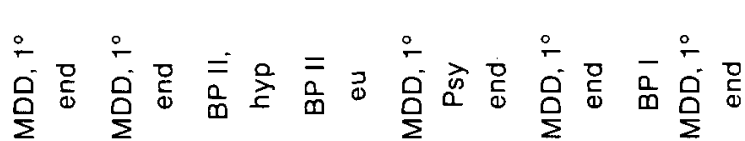

แ $4 \quad$ แ

下 8 \& $\stackrel{\infty}{\infty}$ ஸे

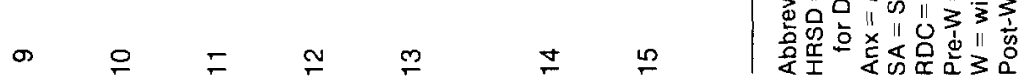


Fig. 1. Highest postdexamethasone plasma cortisol concentrations $(\mu \mathrm{g} / \mathrm{dl})$ for subjects \#1, \#3, \#5, and \#15

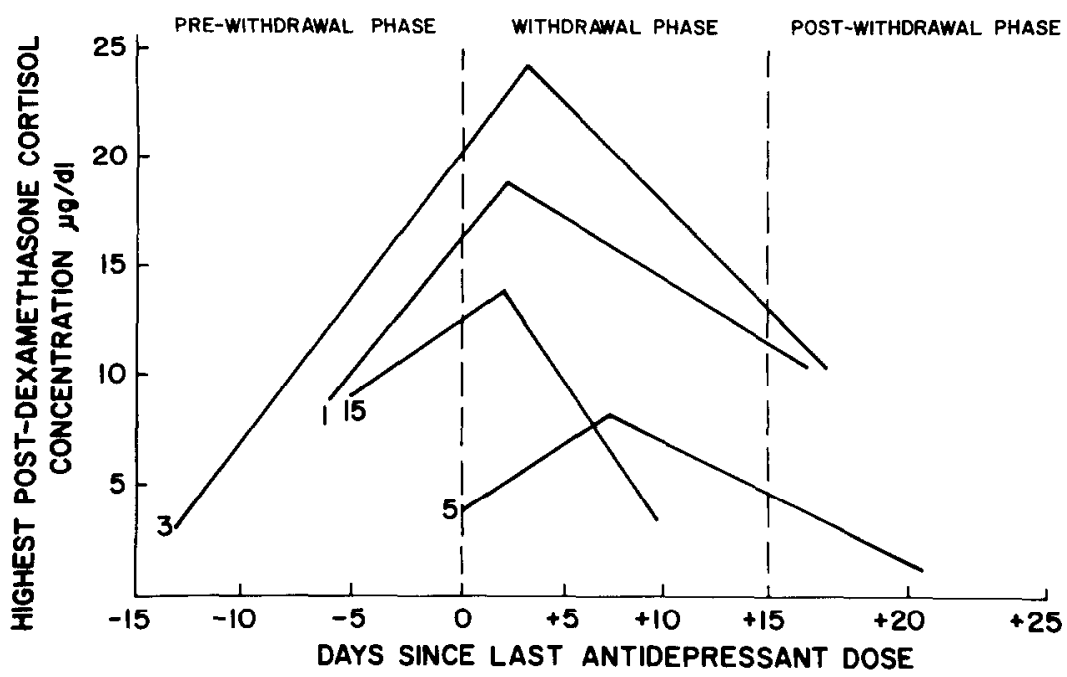

Subjects (\#1,\#3,\#5,\#15, Table 1 ) had three separate DSTs, the first done before antidepressant withdrawal and the second during the drug withdrawal phase. Subjects $\# 1, \# 3$, and $\# 5$ had their third DST done during the postwithdrawal phase, defined as more than 14 days since receiving the last dose of the antidepressant medication. Patient \#15 had both the second and third DSTs during the withdrawal phase.

the severity of depressive symptomatology $(p>>0.1$, Wilcoxon Sign Rank Test, two-tailed).

Fourteen patients had only withdrawal and postwithdrawal DSTs. As shown in Fig. 2, 12 had higher postdexamethasone cortisol levels during the withdrawal phase than the post withdrawal phase ( $p=0.0033, t=3.23, d f=13$, paired $t$ test, one-tailed). Ten of $14(71 \%)$ patients were DST nonsuppressors during withdrawal, whereas 6 of $14(42 \%)$ were nonsuppressors during the postwithdrawal phase. The Fisher's exact probability (one-tailed) was $p=0.03$.

HRSD total scores did not change significantly between the prewithdrawal (mean $\pm \mathrm{SD}=21 \pm 5$ ), withdrawal (mean $\pm \mathrm{SD}=21 \pm 10$ ), and postwithdrawal (mean $\pm \mathrm{SD}=$ $21 \pm 10)$ phases $(p>>0.1$, Mann-Whitney $U$ test, two-tailed, for all statistical comparisons).

To derive an operational measure of stress-anxiety that might be associated with recent hospitalization, we summed the HRSD anxiety (ANX) and somatic anxiety (SA) subscale scores. The sum of these scores did not differ significantly between prewithdrawal $(2.5 \pm 1.5)$, withd rawal $(3.1 \pm 1.9)$, and post withd rawal $(3.4 \pm 2.2)$ phases $(p>>.10$, Mann-Whitney $U$ test, two-tailed).

Fig. 3 portrays DST and clinical rating data for the total samples during each of the three phases. As recorded in lable 1 , weight did not change significantly between the prewithdrawal and postwithd rawal phase DSTs $(p=0.35, t=0.975, d f=14, t$ test on the differences, two-tailed). The highest post-dexamethasone cortisol levels of the 


\section{Fig. 2. Comparisons of highest postdexamethasone cortisol concentrations and rating scale scores obtained during antidepressant withdrawal phase and postwithdrawal phase for 14 subjects}
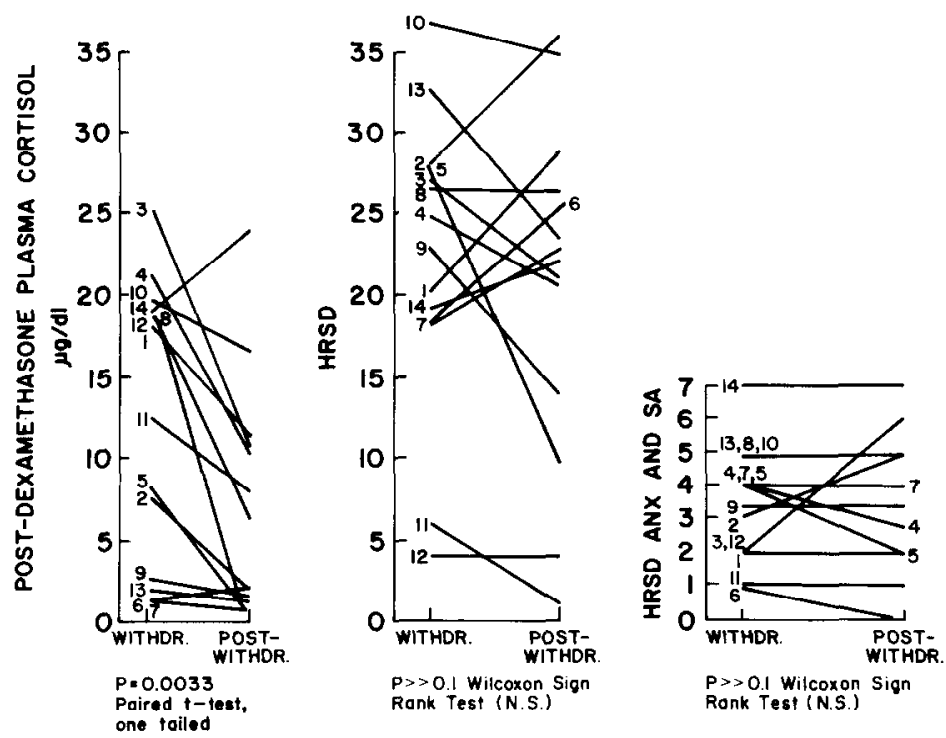

Withdrawal and postwithdrawal phases are defined operationally in the text. The left side of Fig. 2 illustrates the highest postdexamethasone plasma cortisol concentration $\langle\mu \mathrm{g} / \mathrm{d} l\rangle$ for each phase. The middle portion shows corresponding comparisons in total 17-item Hamilton Rating Scalc for Depression (HRSD) scores. The right side summarizes HRSD anxiety (ANX) and somatic (SA) subscale scores for each subject. Statistical comparisons are provided in the text.

withdrawal and postwithdrawal phases did not correlate with weight change $(r=-0.22)$.

The four subjects with simultaneous neuroleptic and antidepressant withdrawal did not differ from the others. The difference in postdexamethasone cortisol level between the withdrawal and postwithdrawal phases for the neuroleptic (mean $\pm \mathrm{SD}=3.92 \pm$ 2.97) and nonneuroleptic (mean $\pm \mathrm{SD}=6.7 \pm 0.35)$ treated subjects $(p=0.48, t=0.74, d f$ =13) was insignificant.

We also analyzed the control group data using two time periods for comparisonthe first 14 days of hospitalization and any time thereafter (Table 2).

The control subjects failed to show the pattern of increased frequency of nonsuppressive DSTs or increases in maximum postdexamethasone cortisol lcvels during the first 2 weeks of hospitalization.

\section{Discussion}

These data suggest that the withdrawal of nonmonoamine oxidase inhibitor antidepressants may produce increases in postdexamethasone plasma cortisol concentrations and an increased prevalence of nonsuppressive DSTs.

The mechanism by which antidepressant withdrawal affects DST nonsuppression is unknown but of potential theoretical importance. Increases in postdexamethasone 
Fig. 3. Prewithdrawal vs. withdrawal; withdrawal vs. postwithdrawal
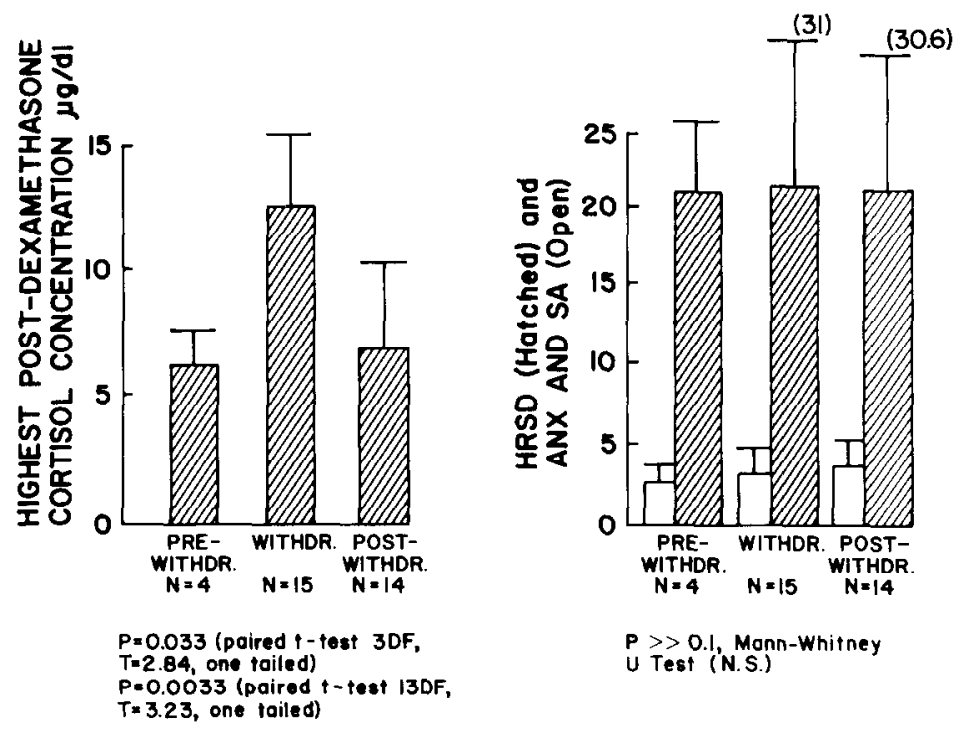

The left side of Fig. 3 portrays highest mean \pm SD postdexamethasone plasma cortisol concentrations ( $\mu \mathrm{g} / \mathrm{dl}$ ! in subjects during prewithdrawal $n=4$ ), withdrawal $n=15 i$, and postwithdrawal $n=14$, phases. The right side illustrates simultaneous clinical ratings from each subject (mean \pm SD) during the 3 phases. The total HRSD score is shown in hatched bars; the sum of anxiety (ANX) and somatic ISA) HRSD subscales is in open bars. Paired $t$ tests were conducted between prewithdrawal and withdrawal phases for the 4 subjects who had DSTs and ratings in both phases, and between withdrawal and postwithdrawal phases for the 14 subjects who had DSTs in both phases. Clinical ratings were compared using the Wilcoxon test.

plasma cortisol concentrations following medication withdrawal might have been due to a worsening of clinical state. Our data did not support this interpretation, however. No patients worsened despite medication withdrawal, suggesting that subjects were unresponsive to treatment, were not treated for adequate periods, or that inadequate time in the drug-free state had passed for them to show significant clinical deterioration. Different time frames might be required to detect neuroendocrine and clinical effects produced by antidepressant discontinuation; thus, these observations are not necessarily incompatible.

These pilot data suggest that the peak in hypothalamic-pituitary-adrenal (HPA) dysregulation occurs between 3 and 14 days after a tricyclic antidepressant is discontinued. This observation is concordant with the known pharmacokinetics of commonly used antidepressants, and with the time course for development of symptoms following antidepressant withdrawal (Dilsaver and Greden, 1984b).

Conceivably, weekly administration of dexamethasone might alter HPA dexamethasone receptor sites. Findings from our semi-control group did not support this, however, nor do prior studies of serial DS Is (Greden et al., 1983; Holsboer et al., 1982), which document moderately good state relationships between serial DSTs and clinical severity. Nevertheless, this question also warrants further prospective study. 


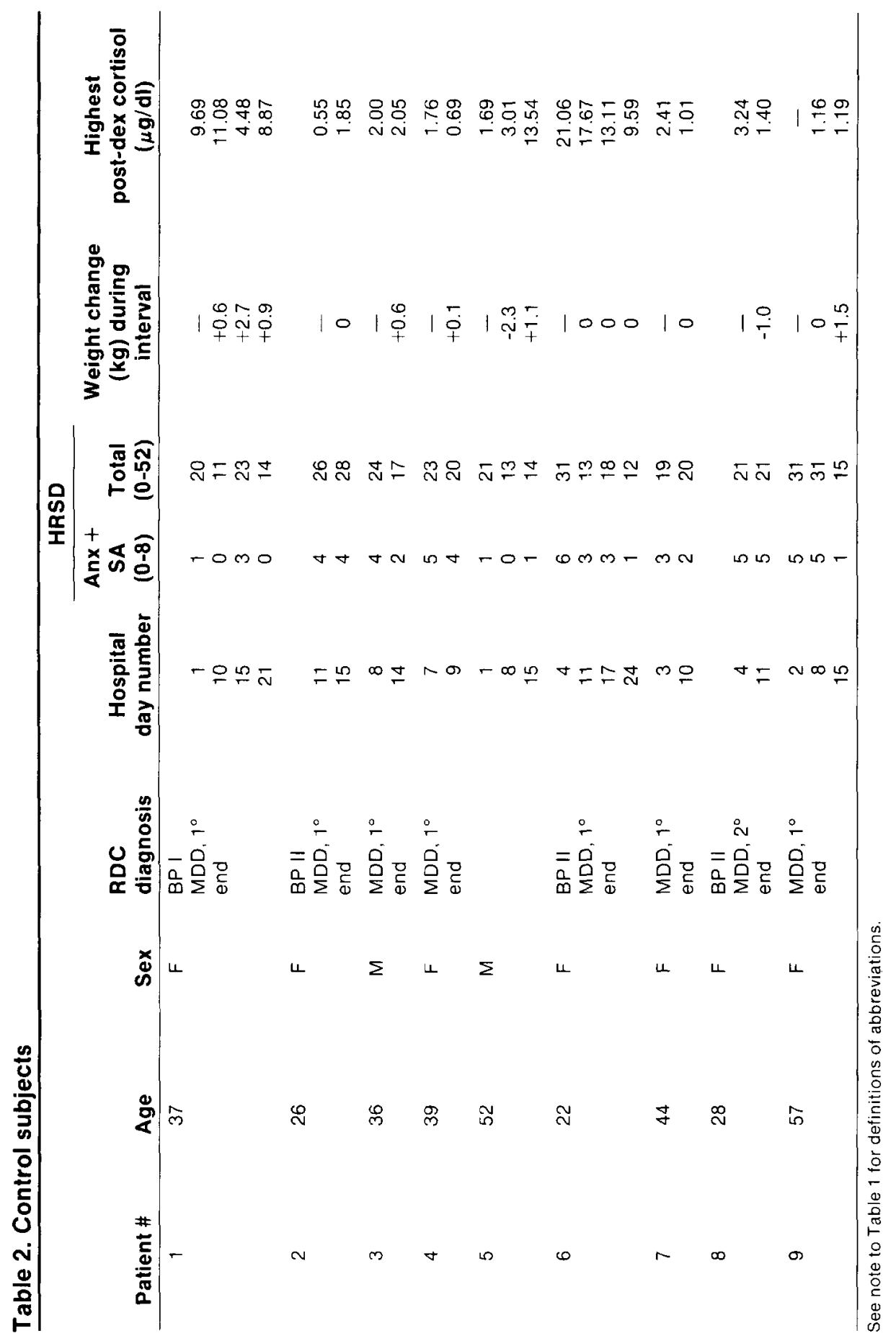


The utility of the DST, like that of other laboratory tests, is diminished if established guidelines for application are not respected. Carroll et al. (1981) emphasized that the value of the DST is maximal when used in a clinical sample at high risk for the presence of endogenous depression, and when it is reasonably certain that the patient does not meet exclusion criteria that interfere with test interpretation. Technical exclusion criteria that invalidate the DST are still being identified. (Privitera et al., 1982; Targum et al., 1982b; Cameron et al., 1983; Kroll et al., 1983). These retrospective pilot data suggest that recent withdrawal of antidepressants may be an exclusion criterion, and should be considered in future research investigations.

Acknowledgment. The authors gratefully acknowledge Mr. Kenneth Guire, Science Research Associate in Biostatistics, School of Public Health, University of Michigan, for his thoughtful assistance.

The research reported was supported, in part, by Public Health Service grant MH-28294; by the Mental Health Research Institute, University of Michigan; and by the State of Michigan Department of Mental Health.

\section{References}

Asfeldt, V.H. Simplified dexamethasone suppression test. Acta Endocrinologica (Copenhagen), 61, 219 (1969).

Berger, M., Krieg, C., and Pirke, K.M. Is the positive dexamethasone test in depressed patients a consequence of weight loss? Neuroendocrinology Letters, 4, 177 (1982).

Berger, M., Pirke, K. M., Doerr, P., Krieg, C., and von Zerssen, D. Influence of weight loss on the dexamethasone suppression test. (Letter to the editor) Archives of General Psychiatry, 40, 585 (1983).

Brown, W.A., Daamen, M., D'Agostino, C., Dockery, E.L., Fournier, P.A., and Parsells, A.H. Cortisol level response to 1- and 2-mg doses of dexamethasone. American Journal of Psychiatry, 140, 609 (1983).

Cameron, O.G., Kronfol, Z., Greden, J.F., and Carroll, B.J. The dexamethasone suppression test in diabetes. Presented at the Annual Meeting of the American Psychosomatic Society, March 24-27, New York (1983).

Carroll, B.J., Feinberg, M., Greden, J.F., Tarika, J., Albala, A.H., Haskett, R.F., James, N.McI., Kronfol, Z., Lohr, N., Steiner, M., de Vigne, J.P., and Young, E. A specific laboratory test for the diagnosis of melancholia. Standardization, validation, and clinical utility. Archives of General Psychiatr.1. 38, 15 (1981).

Carroll, B.J., Greden, J.F., Haskett, R., Feinberg, M., Albala, A., Martin, F.I.R., Ribin, R.T., Heath, B., Sharp, R.T., McLeod, W.L., and McLeod, M.F. Neurotransmitter studies of neuroendocrine pathology in depression: Biogenic Amines Symposium, London, England. Acta Psychiatrica Scandinavica, 61 (Suppl. 280), 183 (1980).

Demers, L. M., and Derck, D.D. Comparison of competitive protein binding analysis and radioimmunoassay for the determination of cortisol in serum and urine. Clinical Biochemistry, 10, 104 (1977).

Dilsaver, S.C., Feinberg, M., and Greden, J.F. Antidepressant withdrawal symptoms treated with anticholinergic agents. American Journal of Psychiatry, 140, 249 (1983a).

Dilsaver. S.C., and Greden. J.F. Antidepressant withdrawal syndromes: Evidence for supersensitivity of the cholinergic system as an etiologic factor. Journal of Clinical Psychopharmacology, 3, 330 (1983).

Dilsaver, S.C., and Greden, J.F. Antidepressant withdrawal induced activation (hypomania and mania) mechanisms and theoretical significance. Brain Research Review, 7, 29 (1984a).

Dilsaver, S.C., and Greden, J.F. Antidepressant withdrawal phenomena: A review. Biological Psychiatry, 19, 237 (1984b). 
Dilsaver, S.C., Kronfol, Z., Sackellares, J.C., and Greden, J.F. Antidepressant withdrawal syndromes: Evidence for the cholinergic overdrive hypothesis. Journal of Clinical Psychopharmacology, 3, 257 (1983b).

Doerr, P., and Berger, M. Physostigmine-induced escape from dexamethasone suppression in normal adults. Biological Psychiatry, 18, 261 (1983).

Edelstein, C.K., Roy-Byrne, P., Fawzy, F.I., and Dornfeld, L. Effects of weight loss on the dexamethasone suppression test. American Journal of Psychiatry, 140, 338 (1982).

Galen, R.S., and Gambino, S.R. Beyond Normality: The Predictive Value and Efficiency of Medical Diagnosis. John Wiley \& Sons, New York (1975).

Goldberg, I.K. Dexamethasone suppression test as indicator of safe withdrawal of antidepressant therapy. Lancet, I, 376 (1980).

Greden, J.F., de Vigne, J.P., Albala, A.A., Tarika, J., Buttenheim, M., Eiser, A., and Carroll, B.J. Serial dexamethasone suppression tests a mong rapidly cycling bipolar patients. Biological Psychiatry, 17, 455 (1982).

Greden. J.F.. Gardner. R.. King, D., Kronfol, 7., and Carroll, B.J. Monitoring antidepressant treatment of melancholia with serial dexamethasone suppression tests: The process of normalization and test-retest reproducibility. Archives of General Psychiatry, 40,493 (1983).

Hamilton, M. A rating scale for depression. Journal of Neurology, Neurosurgery, and Psychiatr., 23, 56 (1960).

Holsboer, F., Liebl, R., and Hofschuster, E. Repeated dexamethasone suppression test during depressive illness. Journal of Affective Disorders, 4, 93 (1982).

Kroll, P., Palmer, C.. and Greden, J.F. The dexamethasone suppression test in patients with alcoholism. Biological Psychiatry, 18, 444 (1983).

Meltzer, H.Y. Cortisol determination and dexamethasone suppression test. Archives of General Psychiatry, 40, 501 (1983).

Murphy, B.E. Some studies of the protein-binding of steroids and their application to the routine micro- and ultra-micro measurement of various steroids in body fluids by competitive protein-binding radioassay. Journal of Clinical Endocrinology and Metabolism, 27, 973 (1967).

Peselow, E.D., Serby, M., Wolkin, A., Deutsch, S.I., Fricchione, G., and Rotrosen, J.P. Plasma cortisol values after dexamethasone in depressed inpatients. Journal of Clinical Ps.'chopharmacolog.', 3, 45 (1983).

Privitera, W.N. Greden, J.F., Gardner, R.W., Ritchie, J.R., and Carroll, B.J. Interference by carbamazepine, with the dexamethasone suppression test. Biological Psychiatry, 17,611 (1982).

Rothschild, A.J., and Schatzberg, A.F. Fluctuating post-dexamethasone cortisol levels in a patient with melancholia. American Journal of Psychiatry, 139, 129 (1982).

Rudorfer, M.V., Hwu, H.G., and Clayton, P.J. Dexamethasone suppression test in primary depression: Significance of family history and psychosis. Biological Psychiatry, 17, 41 (1982).

Rush, A.J., Schlesser, M.A., Giles, D.E., Crowley, C.T., Fairchild, C., and Altschuler, K.Z. The effect of dosage on the dexamethasone suppression test in normal controls. Psychiatry Research, 7, 277 (1982).

Sachar, E.J., Puig-Antich, J., Ryan, N.D., Asnis, G.M., Rabinovich, H., Halpern, F.S. Novacenko, M.S., Ostrow, L., Nelson, B., Davies, M., and Goetz, D. Three tests of cortisol secretion in depression. In: New Research Abstracts, American Psychiatric Association, Washington, DC, NR8 (1983).

Schatzberg. A.F., Rothschild, A.J., Stahl, J.B., Bond, J.C., Rosenbaum, A.H., Lofgren, S.B., MacLaughlin, R.A., Sullivan, M.A., and Cole, J.O. The dexamethasone suppression test: Identification of subtypes of depression. American Journal of Psychiatry, 140, 88 (1983).

Spitzer, R.L., and Endicott, J. Schedule for Affective Disorders and Schizophrenia. New York State Psychiatric Institute, New York (1975).

Spitzer, R.L., Endicott, J., and Robins, E. Research Diagnostic Criteriafor a Selected Group of Functional Disorders. 3rd ed. New York State Psychiatric Institute, New York (1977).

Targum, S.D., Byrnes, S.M., and Sullivan, A.C. Subtypes of unipolar depression distinguished by the dexamethasone suppression test. Journal of Affective Disorders, 4, 21 (1982a). 